\title{
Application of the Extended Kalman Filter for Denoising Movies of the Dynamics of the Dielectric Polarization
}

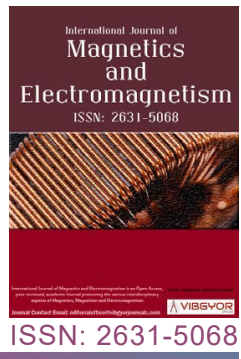

\section{Manfred Fähnle*}

71272 Renningen, Schönblickstraße 95, former member of the Max Planck Institute for Intelligent Systems , Stuttgart, Germany

\begin{abstract}
The extended Kalman filter has been generated to denoise movies of dynamical processes. In the first applications it has been used for systems with only a few degrees of freedom, for instance to denoise the movies of a flying rocket. Later it was suggested for a system with very many degrees if freedom, namely for denoising movies of the fast dissipative magnetization dynamics. The techniques used to produce such movies were the $\mathrm{X}$-ray magnetic circular dichroism (XMCD). There the number $\mathrm{N}$ of degrees of freedom is extremely large, typically $\mathrm{N}$ $=10^{5}$. In this case the calculation of the Jacobian matrix, which occurs in the extended Kalman filter, can not be done numerically, as it was done for systems with few degrees of freedom. The extended Kalman filter had to be speeded up by using a tensorial Green's function for the calculation of the Jacobian. In the present paper it is outlined how such a speeded up version of the Kalman filter can be used also for denoising movies of the dynamics of a system with dielectric polarization. It is also discussed how the tensorial Green's function can be calculated for this case.
\end{abstract}

\section{Description of the Extended Kalman Filter}

A new approach to linear filtering and prediction problems was developed by Kalman [1]. It is used to denoise movies of the dynamics of a quantity, which are often noisy. An example is the denoising of movies of a flying rocket, in which here are only six degrees of freedom (position of the center of gravity, and orientation of the rocket). Later it was suggested to use it for a system with very many degrees of freedom (typically $10^{5}$ ). An example are movies of the fast dissipative magnetization dynamics produced by the $\mathrm{X}$-ray magnetic circular dichroism effect (XMCD) [2]. These are movies, for instance, for the dynamics of a magnetic vortex or of spin waves on time scales of about 100 ps to several ns. The fastest dynamics occurs after exciting a magnetic film by a fs laser pulse. There is an ultrafast demagnetization on the time scale of several $100 \mathrm{fs}$, and a re-magnetization to the original state on a bit longer time scale [3].

The extended Kalman filter works in the following way. We do not repeat to describe the whole procedure and refer to [4] for this., we discuss only the modifications which have to be made for systems with many degrees of freedom. We first describe the work for a magnetic system. The sample is subdivided into $\mathrm{N}$ pixels at positions $\mathbf{r}_{\text {pixel }}$. The state vector $\mathbf{x}\left(t_{k}\right)$ at time $t_{k}$ is given by the vector of

*Corresponding author: Manfred Fähnle, 71272 Renningen, Schönblickstraße 95, former member of the Max Planck Institute for Intelligent Systems, Stuttgart, Germany

Accepted: May 12, 2021; Published: May 14, 2021

Copyright: (c) 2021 Fähnle M. This is an open-access article distributed under the terms of the Creative Commons Attribution License, which permits unrestricted use, distribution, and reproduction in any medium, provided the original author and source are credited.

Fähnle M. Int J Magnetics Electromagnetism 2021, 7:035

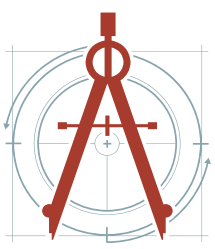


all three components of the magnetization at the pixel points $r_{\text {pixel }}$ at time $t_{k}$. The movies are a least a bit noisy, i.e., $\mathbf{x}\left(t_{k}\right)$ is in part a random variable. The noise arises from the measuring noise and from the process noise. For the XMCD movies the numbers of photons are measured which fall into the detector after transmission of the sample. The probability that an incoming photon is registered is slightly smaller than 1 . This is one reason for the measuring noise. Process noise can appear, for instance, when the considered specimens are structurally inhomogeneous, e.g., because of impurities or because of surface roughness, or when there is thermal noise in the system. The Kalman filter estimates by use of the equation of motion for the considered field variable from the noisy measurement vectors $z\left(t_{k}\right)$ a sequence of non-noisy state vectors $x^{\prime}\left(t_{k}\right)$ which approximate the true state vectors $\mathbf{x}\left(\mathrm{t}_{\mathrm{k}}\right)$ as closely as possible.

In former applications of the Kalman filter (e.g., for the movies of a rocket, see above) systems have been considered for which the exact equation of motion is known (e.g., Newton's equation of motion for the rocket). For the magnetization dynamics the exact equation of motion is not known. It is assumed that the original version of the Landau-Lifschitz-Gilbert equation of motion reference [5] describes the magnetization dynamics satisfactorily. It has been shown reference [4] that this equation is the simplest conceivable of motion but that it has to be extended in several ways to describe accurately all possible situations of the magnetization dynamics. In reference [6] it has been shown that in principle it should nevertheless be possible to use the extended Kalman filter for denoising XMCD movies of the magnetization dynamics. The remaining problem is the explicit calculation of the so-called Jacobian matrix which is used in the prediction part of the extended Kalman filter (see equation 10 of reference [4]) and which has to be determined from the Landau-Lifschitz-Gilbert of motion which is extremely time consuming when a numerical solution of the Landau-Lifschitz-Gilbert is made. For the $10^{5}$ pixel points of the XMCD movies it is strictly impossible to calculate the Jacobian matrix numerically.

The equation of motion for the dynamics of the dielectric polarization is the Debye equation of motion. Again, this is the simplest conceivable equation of motion for the dielectric polarization, which certainly has to be extended in several ways to describe accurately all aspects of the dynamics of the dielectric polarization for all situations. It can be assumed that in principle it should nevertheless be possible to use the extended Kalman filter for denoising movies of the dynamics of the dielectric polarization.

\section{Calculation of the Jacobian Matrix}

The elements of the Jacobian matrix are defined in the following way. We start with a state vector $x\left(t_{k}\right)$ which contains all three components of the magnetizations at the pixel points $r_{\text {pixel }}$. Then the magnetization component $M_{1}$ at the pixel point $r_{i}$ is changed at time $t_{k} a$ bit, and as a result the magnetization component $M_{n}$ at the pixel point $r_{j}$ is changed at time $t_{k+1}$. Formally, this is written as $d M_{1} / d M_{n}\left(r_{j}\right)$ Because there are very small changes, it is possible to linearize the Landau-Lifschitz-Gilbert equation around the starting configuration and to calculate the elements of the Jacobian matrix by a Green's function tensorial method. In reference [7], the method of the tensorial Green's function has been used for the magnetization $\mathbf{M}(\mathbf{r}, \mathbf{t})$ described by a linearized Landau-Lifschitz-Gilbert equation. In general the equations for the components of the tensorial Green's function are very complicated integro-differential equations, especially because of the effects of dipolar couplings. Simplifications arise when considering a situation for which the magnetization in zero field is circular, i.e., is given by a magnetic vortex configuration without perpendicular magnetization components in the vortex core, and when considering discs which are so thin that the magnetization does not depend on the coordinate perpendicular to the disc plane.

Furthermore, it was assumed that there is no macroscopic magnetocrystalline anisotropy in the disc film like in Permalloy films which are often used for experiments of the magnetic vortex dynamics. When neglecting dipolar couplings, then analytical solutions for the components of the tensorial Green's function can be obtained for this situation. For the dynamics of a magnetic vortex in a circular disc the neglect of the perpendicular magnetization components in the vortex center and the neglect of the dipolar fields are approximations. But it can be assumed that the elements of the Jacobian matrix can be calculated with these approximations with satisfactory accuracy, especially for discs with dimensions which are much smaller than the extension of the vortex core. 
Because the calculation of the elements of the Jacobian matrix with the analytical expression is much less time-consuming than their numerical calculation, the use of the tensorial Green's function method makes it possible to use the extended Kalman filter for denoising movies of the fast magnetization dynamics.

In the present paper the use of the extended Kalman filter to denoise movies of the fast dynamics of the dielectric polarization is discussed. One technique for generating such movies is to measure the change of the transmission of the light of a laser probe pulse, resulting from the change of the dielectric polarization by the dynamics of the dielectric polarization [8]. Alternatively, the photon emission electron microscopy (PEEM) can be used [9]. At least in movies produced by the first technique the number of pixel points, i.e., the number of degrees of freedom, is also very large, so that for the use of the extended Kalman filter for denoising the movies it is absolutely necessary to use a Green's tensorial method for the calculation of the Jacobian matric.

The analytic calculation of the tensorial Green's function is given in the appendix of reference [10]. There the tensorial Green's function is calculated analytically for several systems, for instance, the bulk Green's tensor and the Green's tensor for a planar multilayer system. With the use of these analytical results for the tensorial Green's tensor the Jacobian matrix can be calculated very efficiently, and therefore the extended Kalman filter can be used to denoise movies of the fast dynamics of the dielectric polarization.

\section{Conclusion}

In the present paper it is shown that extended Kalman filter can be applied to denoise movies of the dynamics of the dielectric polarization when the Jacobian matrix is calculated analytically by means of the tensorial Green's function method, in spite of the very large number of degrees of free- dom of the dielectric system. Altogether, this is a very interesting extension of the Kalman filter technique which has formally been used, e.g., for denoising movies of the fast magnetization dynamics, to systems with dielectric polarization.

\section{References}

1. Kalman RE (1960) A new approach to linear filtering and prediction problems. J Basic Eng 82: 35-45.

2. Schütz $G$, Wagner $W$, Wilhelm $W$, Kienle $P$, Zeller $R$, et al. (1987) Absorption of circularly polarized $x$ rays in iron. Physical Review Letters 85: 737.

3. Fähnle $M$, Haag $M$, Illg $C$, Mueller $M$, Wang $W$, et al. (2018) Review of ultrafast demagnetization after femtosecond laser pulses: A complex interaction of light with quantum matter. American Journal of Modern Physics 7: 68-74.

4. Kopp M, Harmelin S, Schütz G, Schöllkopf B, Fähnle $M$ (2015) Towards denoising XMCD movies of fast magnetization dynamics using extended Kalman filter. Ultramicroscopy 148: 115-122.

5. Gilbert TL (1956) Formulation, foundations and applications of the phenomenological theory of ferromagnetism. PhD thesis, Illinois Institute of Technology, USA.

6. Fähnle M, Illg C (2011) Electron theory of fast and ultrafast dissipative magnetization dynamics. J Phys Condens Matter 23: 493201.

7. Schweiner F, Fähnle M (2013) Construction of tensorial green's functions for the linearized gilbert equation for magnetization dynamics. IEEE Transactions on Magnetics 49: 2836-2841.

8. Mao SS, Quéré F, Guizard S, Mao X, Russo RE, et al. (2004) Dynamics of femtosecond laser interactions with dielectrics. Applied Physics A 79: 1695-1709.

9. Hofer A, Dunker K, Kiel M, Förster S, Widdra W (2011) Laser-excited PEEM using a fully tunable fs-laser system. Journal of Research and Development 55: 1-8.

10.Buhmann SY (2012) Dispersive forces II. Springer Tracts in Modern Physics, Springer Verlag Berlin, Heidelberg 248: 642. 